\title{
Systems of Equations Satisfied in All Commutative Finite Semigroups
}

\author{
Paweł Parys* \\ Warsaw University \\ parys@mimuw.edu.pl
}

\begin{abstract}
The following problem is considered: check if a system of equations has a solution in every commutative finite semigroup. It is shown that the problem is decidable, and NP-complete. The problem is related with the pumping lemma for regular languages.
\end{abstract}

\section{Introduction}

One of the most famous and deep algorithms existing in formal language theory is Makanin's algorithm [8]. The algorithm takes as an input an system of equations and decides whether the system has a solution in a free semigroup. It has been improved several times. The currently best version of Makanin's algorithm works in EXPSPACE [5] and occupies (including the proof of correctness) over forty pages. Recently new algorithms to decide solvability of general word equations, using different ideas, have been found [1110]. The algorithm in [10] works in PSPACE.

A related problem is to check if a system of equations is satisfied in all semigroups simultaneously. However, it can be easily shown that a system of equations having a solution in the free semigroup, also has a solution in every other semigroup. In this sense, for equations the free semigroup is the most difficult of semigroups. This argument, however, fails for finite semigroups. There are systems, which have solutions in all finite semigroups, but not in the free semigroup. For instance in a finite semigroup, when we add an element to itself several times, we always start looping, which can cause the existence of a solution. It's easy to show that in every finite semigroup there exists an idempotent element (i.e. a solution of an equation $x \cdot x=x$ ). It is enough to take any element and to add it to itself appropriate number of times. This fails in the free semigroup (no empty words). Solving of other equations will be a generalization of this observation.

This paper is devoted to solving equations over finite semigroups. Formally the following problem may be considered: Given a system of equations (with variables and coefficients), decide if the system has a solution in every finite semigroup and for every evaluation of the coefficients in this semigroups. Intuitively speaking, an opponent chooses a semigroup and values of coefficients and we have to show values of variables such that the equations will be satisfied.

\footnotetext{
^ Author supported by Polish government grant no. N206 008 32/0810.
} 
For example consider the following system of equations:

$$
\left\{\begin{array}{c}
x \cdot x=x \\
a \cdot y \cdot b=x
\end{array}\right.
$$

In every finite semigroup and for every values of coefficients $a$ and $b$, we want to have such values of variables $x$ and $y$ that the system is satisfied. In other words we need such an idempotent $x$, which ,,begins" with $a$ and ,,ends" with $b$. One can easily prove that such an idempotent exists, regardless of the choice of the semigroup and the coefficients $a$ and $b$.

Checking if a system has a solution in every finite semigroup is interesting itself, but it has also some motivation. There is a correspondence between finite semigroups and regular languages, so questions about semigroups are also questions about regular languages. Solving equations in finite semigroups can be seen as a generalization of pumping. What does it mean that a system of equations has a solution in every finite semigroup? For any finite semigroup (finite automaton) we choose, there would exist such values of the variables such that the left and right sides evaluate to the same element. So using these values of variables we will deceive any semigroup: it cannot distinguish between the left and right side. This idea was used in recent work over tree-walking automata, where standard pumping lemmas proved to be inadequate, and nonexpressiveness results were shown by using equations in semigroups. For example in [1, for every semigroup and for every $a$ and $b$ they need to have $u$ and $v$ such that $u=u \cdot a \cdot u=u \cdot b \cdot v$ and $v=v \cdot a \cdot u=v \cdot b \cdot v$.

In this paper I concentrate on commutative semigroups. I show that solving equations in all finite commutative semigroups is not only decidable, but NPcomplete.

Theorem 1.1. The following problem is NP-complete: Given a system of word equations, decide if the system has a solution in every finite commutative semigroup.

There is the following easy extension of that theorem (proved in Section 4.4).

Corollary 1.2. The following problem is decidable: Given a closed positive $\Pi_{2}$ formula (i.e. of the form $\forall \ldots \forall \exists \ldots \exists$ (positive sentence) where elementary sentences are equations) decide if it is true in every finite commutative semigroup.

The non-commutative case is left open. Some preliminary results are described in Section 6 .

Related work. Here we ask if for every choice of coefficients there exist values of variables. A natural extension of this question is deciding whether an arbitrary first-order formula is satisfied in any finite semigroup. The problem is undecidable [4, even for very special case of formulas. For commutative finite semigroups the problem was not considered yet, to the best of the author's knowledge. Checking if a first-order formula is satisfied in a free semigroup is undecidable [12. 
However for a commutative free semigroup the problem is decidable, since it can be encoded in well known Presburger arithmetic.

Another connected problem is solving a system of equations in a given finite semigroup 7 .

\section{Notations and Definitions}

Vectors of letters like $c_{1}, \ldots, c_{m}$ or $X_{1}, \ldots, X_{n}$ etc. will be denoted by $\bar{c}, \bar{X}, \ldots$ When I need to consider simultaneously several vectors of the same type, I use superscripts: $\bar{X}^{(1)}, \ldots, \bar{X}^{(h)}, \ldots$

As we have only commutative semigroups I use plus sign for describing the semigroup operation. For $a$ in a semigroup and $k \geq 1 \mathrm{I}$ write $k \cdot a$ for $a$ added to itself $k$ times.

I fix the following finite nonempty alphabets:

$\Sigma_{0}=\left\{X_{1}, \ldots, X_{\gamma}\right\}$ - the alphabet of variables,

$\Sigma_{1}=\left\{C_{1}, \ldots, C_{\omega}\right\}$ - the alphabet of coefficients.

An interpretation of coefficients in a semigroup $S$ is a vector $\bar{c}=\left(c_{1}, \ldots, c_{\omega}\right)$ of elements of $S$ (an element $c_{i}$ corresponds to a coefficient $C_{i}$ ). It is easier to treat an interpretation of coefficients as a part of a semigroup. A pair $(S, \bar{c})$ of a semigroup and an interpretation of coefficients in it will be called a semigroup with coefficients. Since now as a semigroup I will understand a semigroup with coefficients and sometimes I will simply write $S$ for $(S, \bar{c})$.

A system of equations $\bar{\phi}$ is a system of equalities of the form

$$
\left\{\begin{array}{c}
\phi_{11}=\phi_{12} \\
\cdots \\
\phi_{m 1}=\phi_{m 2}
\end{array}\right.
$$

where $\phi_{11}, \ldots, \phi_{m 1}, \phi_{12}, \ldots, \phi_{m 2}$ are nonempty words over $\Sigma_{0} \cup \Sigma_{1}$. Sometimes I will write plus signs between symbols in the equations (just for convenience). For a given semigroup with coefficients $(S, \bar{c})$ and vector $\bar{x}$ of elements of $S$, by $\phi_{j s}(\bar{x})$ I denote evaluation of $\phi_{j s}$ in semigroup $S$ with $c_{i}$ (and $x_{i}$ ) substituted for $C_{i}$ (and $\left.X_{i}\right)$. For a given semigroup with coefficients $(S, \bar{c})$, a solution of the system $\bar{\phi}$ is a vector $\bar{x} \in S$ such that $\phi_{i 1}(\bar{x})=\phi_{i 2}(\bar{x})$ for all $1 \leq i \leq m$.

A homomorphism of semigroups with coefficients from $(S, \bar{c})$ to $\left(S^{\prime}, \bar{c}^{\prime}\right)$ is a homomorphism of semigroups $f: S \rightarrow S^{\prime}$ such that $f\left(c_{i}\right)=c_{i}^{\prime}$ for every $i$. See that if a system $\bar{\phi}$ has a solution $\bar{x}$ in $(S, \bar{c})$ and we have any homomorphism $f:(S, \bar{c}) \rightarrow\left(S^{\prime}, \bar{c}^{\prime}\right)$, then image of $\bar{x}$ is obviously a solution in $\left(S^{\prime}, \bar{c}^{\prime}\right)$.

We will shortly say that a system has a solution in the class FinComm if for every commutative finite semigroup with coefficients there exists a solution of the system. Note the quantifier alternation: for all semigroups and all values of the coefficients, one must be able to find a values of the variables that yield a solution. In the article I present an algorithm for solving the following problem:

Input: a system of equations.

Output: Has the system a solution in every commutative finite semigroup with coefficients? 
What would happen if we've skipped the word "finite"? When a system has a solution in every commutative semigroup it also has in a free commutative semigroup. There is a homomorphism from the free commutative semigroup to any other, so from a solution in free commutative semigroup we get a solution in any other commutative semigroup. Therefore the problem would reduce to finding solutions in the free commutative semigroup, which is easy.

\section{Special Form of Equations: Variables on Both Sides}

Definition 3.1. I will say that an equation is balanced if on its every side there is at least one variable. I will say that a system of equations is balanced if every equation is balanced.

The problem is somehow easier if we consider only balanced systems. At the beginning I will solve the problem in this special case. The results from this section will be used later for solving the general case.

\subsection{One Coefficient}

At the very beginning we will consider an even simpler form of systems, where at most one coefficient is used in the system. A general balanced system will be later reduced to several such systems. The following two theorems tell us that it is sufficient to solve such systems over $\mathbb{Z}$.

Theorem 3.2. Let $C$ be a coefficient and let $\bar{\phi}$ be a balanced system where no coefficients other than $C$ appear in the system. Then the following statements are equivalent:

1. the system $\bar{\phi}$ has a solution in Fin Comm;

2. for every $n \geq 2$ the system $\bar{\phi}$ has a solution in the group $\mathbb{Z}_{n}$ (integers modulo n) with an interpretation $C \mapsto 1$.

Interpretation of coefficients other than $C$ doesn't matter, as they do not appear in the system, but for completeness we should fix it somehow. Condition 1 above could be replaced by the assertion for all finite semigroups, not only commutative (we do not use commutativity in the proof below).

In a proof of the theorem I will use the following fact:

Fact 3.3. For a given element $c$ of a finite semigroup $S$ there exists $N$ such that $2 N \cdot c=N \cdot c$.

Proof. Look at all multiples of $c$. As there is only finite number of elements in $S$, there have to be $k \cdot c=(k+l) \cdot c$ for some $k, l \geq 1$. Adding to this equation $l \cdot c$ several times we get

$$
k \cdot c=(k+l) \cdot c=(k+2 l) \cdot c=\ldots=(k+k l) \cdot c .
$$

Then adding $(l-1) k \cdot c$ to it we get $k l \cdot c=2 k l \cdot c$. So taking $N=k l$ we are done. 
Proof (of Theorem [3.2). 1 $\Rightarrow$ 2. Obvious, because 2 is a special case of 1 .

$11 \Leftarrow 2$. Fix a commutative finite semigroup $S$ with an interpretation $c \in S$ of the coefficient $C$, for which the system should have a solution. Let $N$ be such that $2 N \cdot c=N \cdot c$ (from fact 3.3 ). We have the following two properties for every $k, l \geq 0$ :

- it holds $(N+k) \cdot c+(N+l) \cdot c=(N+k+l) \cdot c$;

- if additionally $k \equiv l \quad(\bmod N)$ it holds $(N+k) \cdot c=(N+l) \cdot c$.

Let $\bar{y}$ will be a solution of the system in $\mathbb{Z}_{n}$ (understood as numbers from 0 to $N-1)$, which exists from point 2. We take $x_{j}=\left(N+y_{j}\right) \cdot c$ for all $1 \leq j \leq \gamma$. Then for every $1 \leq i \leq m, s=1,2$ we have $\phi_{i s}(\bar{x})=\left(N+\phi_{i s}(\bar{y})\right) \cdot c$. Since $\bar{y}$ is a solution in $\mathbb{Z}_{n}$, we have $\phi_{i 1}(\bar{y}) \equiv \phi_{i 2}(\bar{y}) \quad(\bmod N)$, so $\left(N+\phi_{i 1}(\bar{y})\right) \cdot c=\left(N+\phi_{i 2}(\bar{y})\right) \cdot c$, which means that $\bar{x}$ is a solution in $S$.

Theorem 3.4. Let $C$ be a coefficient and let $\bar{\phi}$ be a balanced system where no coefficients other than $C$ appear in the system. Then the following statements are equivalent:

1. for every $n \geq 2$ the system $\bar{\phi}$ has a solution in the group $\mathbb{Z}_{n}$ with an interpretation $C \mapsto 1$;

2. the system $\bar{\phi}$ has a solution in the group $\mathbb{Z}$ with an interpretation $C \mapsto 1$.

Proof. 1$] \Leftrightarrow 2$. This implication is almost obvious. For every $n$ there is a homomorphism from $\mathbb{Z}$ to $\mathbb{Z}_{n}$ (taking numbers modulo $n$ ), so if we have a solution in $\mathbb{Z}$, we also have it in $\mathbb{Z}_{n}$.

$1 \Rightarrow 2$. In this theorem in fact we deal with classical systems of number equations in $\mathbb{Z}$ or $\mathbb{Z}_{n}$. The system can be written in the form of $\overline{\bar{a}} \cdot \bar{X}=\bar{b}$ where $\overline{\bar{a}}$ and $\bar{b}$ are a matrix and a vector of integer numbers and $\bar{X}$ is a vector of variables. For solving this system in $\mathbb{Z}$ we can perform a Gauss elimination on the pair $(\overline{\bar{a}}, \bar{b})$. To keep all the constants in $\mathbb{Z}$ we cannot divide a equation by a number, we can only multiply, but it is enough. The only difference from normal Gauss elimination (with division allowed) is that we are not able to get ones "on the diagonal", we get there arbitrary nonzero numbers. After possibly changing numeration of variables (order of columns in $\overline{\bar{a}}$ ) we get the following equivalent system

$$
\left[\begin{array}{ccccccccc}
a_{11} & 0 & 0 & \ldots & 0 & a_{1, k+1} & a_{1, k+2} & \ldots & a_{1 \gamma} \\
0 & a_{22} & 0 & \ldots & 0 & a_{2, k+1} & a_{2, k+2} & \ldots & a_{2 \gamma} \\
0 & 0 & a_{33} & \ldots & 0 & a_{3, k+1} & a_{3, k+2} & \ldots & a_{3 \gamma} \\
\vdots & \vdots & \vdots & \ddots & \vdots & \vdots & \vdots & \ddots & \vdots \\
0 & 0 & 0 & \ldots & a_{k k} & a_{k, k+1} & a_{k, k+2} & \ldots & a_{k \gamma} \\
0 & 0 & 0 & \ldots & 0 & 0 & 0 & \ldots & 0 \\
\vdots & \vdots & \vdots & \ddots & \vdots & \vdots & \vdots & \ddots & \vdots \\
0 & 0 & 0 & \ldots & 0 & 0 & 0 & \ldots & 0
\end{array}\right]\left[\begin{array}{c}
X_{1} \\
X_{2} \\
X_{3} \\
\vdots \\
X_{k} \\
X_{k+1} \\
X_{k+2} \\
\vdots \\
X_{\gamma}
\end{array}\right]=\left[\begin{array}{c}
b_{1} \\
b_{2} \\
b_{3} \\
\vdots \\
b_{k} \\
b_{k+1} \\
\vdots \\
b_{m}
\end{array}\right]
$$

where $a_{11}, \ldots, a_{k k}$ are nonzero. 
The same operations we can do, when we are considering the system over $\mathbb{Z}_{n}$, and we get the same system (with the exception that all numbers are treated modulo $n$ ). Here the system we get is not necessarily equivalent to the original one (when multiplying by a number which has common divisors with $n$, a equality may become true, when it wasn't). But if the original system had a solution, then this also has (for every $\mathbb{Z}_{n}$ ).

Firstly see that $b_{k+1}=b_{k+2}=\ldots=b_{m}=0$. Otherwise for $b_{i} \neq 0$ we have a equation $0=b_{i}$ which should be satisfied in all semigroups $\mathbb{Z}_{n}$, but is not satisfied in almost all of them, e.g. for any $n>b_{i}$.

Let $n=a_{11} \cdot a_{22} \cdot \ldots \cdot a_{k k}$. Let $x_{1}, \ldots, x_{\gamma}$ be a solution in this $\mathbb{Z}_{n}$. The $i$-th equation $(1 \leq i \leq k)$ says that

$$
a_{i i} x_{i}+a_{i, k+1} x_{k+1}+a_{i, k+2} x_{k+2}+\ldots+a_{i \gamma} x_{\gamma} \equiv b_{i} \quad\left(\bmod a_{11} \cdot a_{22} \cdot \ldots \cdot a_{k k}\right)
$$

from which we have

$$
a_{i i} x_{i}+a_{i, k+1} x_{k+1}+a_{i, k+2} x_{k+2}+\ldots+a_{i \gamma} x_{\gamma} \equiv b_{i} \quad\left(\bmod a_{i i}\right)
$$

which means that

$$
a_{i i} \mid\left(b_{i}-a_{i, k+1} x_{k+1}-a_{i, k+2} x_{k+2}-\ldots-a_{i \gamma} x_{\gamma}\right)
$$

As a solution for $\mathbb{Z}$ we will take:

$$
x_{i}^{\prime}=\left\{\begin{array}{lr}
\left(b_{i}-a_{i, k+1} x_{k+1}-a_{i, k+2} x_{k+2}-\ldots-a_{i \gamma} x_{\gamma}\right) \cdot \frac{1}{a_{i i}} & \text { for } 1 \leq i \leq k \\
x_{i} & \text { for } k+1 \leq i \leq \gamma
\end{array}\right.
$$

Condition (11) guarantees that $x_{i}^{\prime}$ is integer. It's easy to see that it is really a solution.

\subsection{Many Coefficients}

Definition 3.5. For a given balanced system $\bar{\phi}$ and a coefficient $C \in \Sigma_{1}$ we define a projection $\bar{\phi}^{(C)}$ as a system obtained from $\bar{\phi}$ by erasing all coefficients other than $C$.

Notice that the assumption that $\bar{\phi}$ has a variable on every side (is balanced) guarantees that $\bar{\phi}^{(C)}$ also has a variable on every side. In particular the sides are nonempty, so we get a well defined system.

Lemma 3.6. Let $\bar{\phi}$ be a balanced system. Then the following statements are equivalent:

1. the system $\bar{\phi}$ has a solution in Fin Comm;

2. for every coefficient $C \in \Sigma_{1}$ and $n \geq 2$ the system $\bar{\phi}^{(C)}$ has a solution in the group $\mathbb{Z}_{n}$ with an interpretation $C \mapsto 1$. 
Proof. 1 $\Rightarrow$ 2. Almost obvious. Fix $C$ and $n$. As a special case of 1 we get that the system $\bar{\phi}$ has a solution in $\mathbb{Z}_{n}$ with interpretation $C \mapsto 1$ and $D \mapsto 0$ for all $D \in \Sigma_{1}, D \neq C$. But this solution is also a solution of $\bar{\phi}^{(C)}$, because evaluation of sides of $\bar{\phi}$ and $\bar{\phi}^{(C)}$ are the same (the only difference is that in $\bar{\phi}$ we add 0 several times).

$1 \Leftarrow 2$ Fix a commutative finite semigroup $S$ with coefficients $\bar{c}$. From 2 and Theorem 3.2 for every $C_{i} \in \Sigma_{1}$ we have a solution $\bar{x}^{\left(C_{i}\right)}$ of the projection $\bar{\phi}^{\left(C_{i}\right)}$ in $S$. As our solution of the whole system we take the sum of these solutions: $x_{j}=x_{j}^{\left(C_{1}\right)}+x_{j}^{\left(C_{2}\right)}+\ldots+x_{j}^{\left(C_{\omega}\right)}$ for every $1 \leq j \leq \gamma$. Then the evaluation of a side of an equation will be the sum of evaluations of sides of equations for one coefficient: $\phi_{k s}(\bar{x})=\phi_{k s}^{\left(C_{1}\right)}\left(\bar{x}^{\left(C_{1}\right)}\right)+\phi_{k s}^{\left(C_{2}\right)}\left(\bar{x}^{\left(C_{2}\right)}\right)+\ldots+\phi_{k s}^{\left(C_{\omega}\right)}\left(\bar{x}^{\left(C_{\omega}\right)}\right)$. This is because every $c_{i}$ or $x_{j}^{\left(C_{i}\right)}$ appears the same number of times in $\phi_{k s}(\bar{x})$ as in $\phi_{k s}^{\left(C_{i}\right)}\left(\bar{x}^{\left(C_{i}\right)}\right)$ and does not appear in any other element. Of course we've used the assumption that the semigroup is commutative.

As an immediate corollary of Lemma 3.6 and Theorem 3.4 we get the following theorem:

Theorem 3.7. Let $\bar{\phi}$ be a balanced system. Then the following statements are equivalent:

1. the system $\bar{\phi}$ has a solution in FinComm;

2. for every coefficient $C \in \Sigma_{1}$ the system $\bar{\phi}^{(C)}$ has a solution in the group $\mathbb{Z}$ with an interpretation $C \mapsto 1$.

So we've got an easy to check criterion for testing if such system has a solution in the class FinComm.

\section{General Case}

\subsection{Everywhere Something Is One Everywhere}

Now I will prove an important technical lemma, which simplifies further argumentation. Here we need a version of the lemma for commutative finite semigroups, but the same lemma is true for general finite semigroups or for any variety.

Lemma 4.1. Let $\left\{\bar{\phi}^{(1)}, \ldots, \bar{\phi}^{(N)}\right\}$ be systems of equations such that in every commutative finite semigroup with coefficients some $\bar{\phi}^{(i)}$ has a solution. Then some of the systems $\bar{\phi}^{(i)}$ has a solution in every of these semigroups.

Proof. Assume that for every system $\bar{\phi}^{(i)}$ there exists a semigroup $S_{i}$ in which there is no solution of $\bar{\phi}^{(i)}$. Look at the product semigroup $S=S_{1} \times \ldots \times$ $S_{N}$ (naturally interpretation of coefficient in the product is a sequence of its interpretations in every $S_{i}$ ). Some system $\bar{\phi}^{(i)}$ has to have a solution in $S$. But we have a homomorphism from $S$ to $S_{i}$ (a projection), so $\bar{\phi}^{(i)}$ has a solution in $S_{i}$ too. But we've assumed that it hasn't, we've got contradiction, so the theorem is true. 


\subsection{Removing "Wrong" Variables}

Let $\bar{\phi}$ be a system in which in some equations on one side there are only coefficients and on the other side there are also variables. We will replace the system by a number of simpler systems. For notational simplicity, assume that this happened in the first equation: that on the right side of this equation $\left(\phi_{12}\right)$ there are only coefficients, and that on its left side $\left(\phi_{11}\right)$ there is at least the variable $X_{1}$. For a word of coefficients $w \in \Sigma_{1}^{*}$ we define a system $\bar{\phi}^{(w)}$. It will be $\bar{\phi}$ in which we replace every occurrence of $X_{1}$ in every equation by the word $w$. We will be considering these systems for all nonempty subsequences of the right side of the first equation (in fact the order of coefficients in $w$ doesn't matter, as we have only commutative semigroups, so we can also think about all submultisets of the $\left.\phi_{12}\right)$. Obviously there are only finitely many of these subsequences.

Theorem 4.2. For a system $\bar{\phi}$ as above, the system has a solution in FinComm if and only if for some $w$ - subsequence of $\phi_{12}$, the system $\bar{\phi}^{(w)}$ has a solution in FinComm.

Proof. $\Leftarrow$. Let $w$ be this subsequence of $\phi_{12}$, for which $\phi^{(w)}$ has a solution in FinComm. Fix a commutative finite semigroup with coefficients $S$, for which we need a solution of $\bar{\phi}$. Let $\bar{x}$ be a solution of $\bar{\phi}^{(w)}$ there. As a solution of $\bar{\phi}$ we can take an evaluation of $w$ as $x_{1}$ and values from $\bar{x}$ as the other variables. Then the evaluation of sides of $\bar{\phi}$ and $\bar{\phi}^{(w)}$ are the same, so this is a solution of $\bar{\phi}$ (the only difference between $\bar{\phi}$ and $\bar{\phi}^{(w)}$ is that on the places of $X_{1}$ we have $w$, but they both evaluates to the same value).

$\Rightarrow$. According to Lemma 4.1 it is enough to show that in every commutative finite semigroup for every interpretation of coefficients at least one of these systems has a solution. Then the lemma would say that one of the systems would have a solution in every of these semigroups.

Fix a commutative finite semigroup with coefficients $S$, for which we need a solution of some system $\bar{\phi}^{(w)}$. We will construct a semigroup $S^{\prime}$ and basing on a solution of $\bar{\phi}$ in it, we will construct a solution of some $\bar{\phi}^{(w)}$ in $S$. Let $N$ be the length of $\phi_{12}$ (the right side of the first equation, which contains only coefficients). The semigroup $S^{\prime}$ will contain two disjoint parts:

1. all the elements of $S$;

2. all commutative words (multisets) of coefficients from $\Sigma_{1}$ up to length $N$.

Now we need to define an operation. Inside $S$ we just add in $S$. When adding between the parts, we evaluate the word of coefficients in $S$ and then add in $S$. When adding two words of coefficients, we concatenate it. If the result fits into second part (has length $\leq N$ ), we just take it. When it is longer, we evaluate it in $S$. The idea is that $S^{\prime}$ for short words simulates free commutative semigroup. Words no longer than $N$ we remember as they are, for longer words we remember only their value in $S$.

We take an interpretation of coefficients in $S^{\prime}$ such that a coefficient is interpreted as an one-letter word containing it. $S^{\prime}$ is a commutative finite semigroup, so $\bar{\phi}$ has a solution $\bar{x}^{\prime}$ in it. See that when something is in the first part, then 
after adding anything it will remain in this part. The right side of the first equation, which contains just $N$ coefficients, in $S^{\prime}$ will evaluate to itself in the second part. This means that $x_{1}^{\prime}$ (and the whole left side) is also in the second part. Moreover, $x_{1}^{\prime}$ is a submultiset of the right side. We will take $x_{1}^{\prime}$ as the word $w$. Now we need to have a solution of $\bar{\phi}^{(w)}$ in $S$. As $x_{i}$ (for $2 \leq i \leq \gamma$ ) we will take $x_{i}^{\prime}$ when it is in the first part or evaluation of $x_{i}^{\prime}$ in $S$, when it is in the second part. It's easy to see that $\bar{\phi}^{(w)}$ gives now the same equalities, as $\bar{\phi}$ before (when an equation from $\bar{\phi}$ in $S^{\prime}$ has given an equality on words, then it also holds after evaluation to $S$ ). So it's a solution in $S$ and we're done.

\subsection{The Algorithm}

Theorem 4.2 almost gives us an algorithm. In every moment we will have a set of systems, about which we'd like to know if at least one of the systems has a solution in FinComm. At the very beginning the set contains only the original system. Then we repeatedly replace a system, in which in some equations variables are only on one side, by a number of systems, as described above. See that after such step, we get systems containing less variables, so the process has to finish. At the end we get a set of systems, in which every equation contains at least one variable on both sides or no variables at any side.

Now see what happens, if in an equation there are only coefficients. If the number of coefficients of every kind is equal on both sides (the sides are equal as multisets), then the equation is always satisfied, because our semigroups are commutative. In this case we can just remove this equation and we get equivalent system. Otherwise there is an coefficient $C$, which on left side appears $k$ times and on the right side $l$ times, where $k \neq l$. Such equation is not satisfied in FinComm, it is even very unlikely to be satisfied in any commutative finite semigroup. For example consider an additive group $\mathbb{Z}_{k+l+1}$ with interpretation $C \mapsto 1$ and $D \mapsto 0$ for all $D \in \Sigma_{1}, D \neq C$. Here left side evaluates to $k$ and right side to $l$, which aren't equal, so the equality isn't satisfied. This means that we can immediately say that system containing such equation cannot have a solution in FinComm.

Finally we get a set of balanced systems; we want to say if any of them has a solution in FinComm. But for such systems it can be determined by the algorithm from section 3

\subsection{Positive $\Pi_{2}$ Formulas}

We will now prove Corollary 1.2. Here on the input we have a closed positive $\Pi_{2}$ formula instead of a system of equations. In fact original Theorem 1.1 (and the above algorithm) solves a situation, when only conjunctions are used. A formula with disjunctions can be normalized to a form saying that (in every commutative semigroup) for every choice of coefficients there exist values of variables such that some of given systems is satisfied. In the light of Lemma 4.1 we can equivalently say that some of these systems is satisfied in FinComm. So it is enough to check each of these systems separately using above algorithm - if one of them 
is satisfied in FinComm, then the formula is true in every commutative finite semigroup.

However during the mentioned normalization, the size of a formula can grow exponentially, so we lose the NP complexity.

\section{Complexity}

In this section I will show that the problem is NP-complete. To talk about the complexity we need to know how we represent the system and how is its size defined. The easiest (but not good enough) way is to remember an equation as it is, as a list of coefficients and variables. When we use this representation, then during the operation of our algorithm the size of the system can grow exponentially, so it wouldn't work in NP. But we can do better: a side of a equation will be described by numbers of occurrences of every symbol (coefficient or variable). So when a symbol repeats $N$ times, the binary representation of $N$ will be remembered, which has a length of $\Theta(\log N)$.

Firstly I will show that the problem is in NP. Of course I will use the algorithm described above in the paper. Essentially the algorithm consists of two parts. In the first part we remove a variable from unbalanced equation several times and substitute for it some subsequence of the other side of the equation (see subsection 4.2). Note that in nondeterministic model we can just guess the correct substitution instead of checking all the possibilities. In the second part we solve a balanced system of equations over $\mathbb{Z}$. This can be done in NP (see [2, the solution will have polynomial size, so we can just guess it).

The only question is what is the length of the system after the first part. We need to show that it will grow at most polynomially. Let $V_{i}$ and $B_{i}$ be a total number of occurrences of variables and of coefficients in the system after the $i$-th step of the algorithm (in particular $V_{0}$ and $B_{0}$ are these numbers in the initial system). Note that the length of the initial system is at least $\Omega\left(\log \left|V_{0}+B_{0}\right|\right)$. In $i$-th step we substitute for a variable at most $B_{i-1}$ coefficients, and the variable occurs at most $V_{i-1}$ times, so

$$
B_{i} \leq B_{i-1}+V_{i-1} B_{i-1}=\left(V_{i-1}+1\right) B_{i-1}
$$

The number of occurrences of variables even decrease, so $V_{i} \leq V_{i-1}$. After $k$ steps we have: $V_{k} \leq V_{0}$ and $B_{k} \leq\left(V_{0}+1\right)^{k} B_{0}$. Let $N$ be the length of the initial system, $\gamma \leq N$ - number of different variables and $\omega \leq N$ - number of different coefficients. In each step we remove one variable, so there are at most $k \leq \gamma \leq N$ steps. In the resulting system we have $2 m$ sides of $m$ equations, every of which needs length at most $\gamma \log V_{k}$ for describing variables and $\omega \log B_{k}$ for describing coefficients. So the length of the resulting system will be at most:

$$
\begin{aligned}
2 m\left(\gamma \log V_{k}+\omega \log B_{k}\right) & \leq 2 N^{2}\left(\log V_{0}+\log \left(\left(V_{0}+1\right)^{N} B_{0}\right)\right)= \\
& =2 N^{2}\left(\log V_{0}+N \log \left(V_{0}+1\right)+\log B_{0}\right) \leq \\
& \leq O\left(N^{3}\right) O\left(\log \left|V_{0}+B_{0}\right|\right) \leq O\left(N^{4}\right)
\end{aligned}
$$

so it's polynomial. 
Now I will show that the problem is NP-hard. To do that I will reduce to it the NP-complete clique problem (defined in [6]). Almost the same proof would also work for a problem of solving systems of equations in the commutative free semigroup. Assume that we are looking for a clique of size $k$ in a graph $G=(V, E)$. Assume that $V=\{1,2, \ldots,|V|\}$. We will have only one coefficient $C$. We will have a variable $X_{i}$ for each vertex and helper variables $X_{i}^{\prime}$ and $X_{i j}^{\prime \prime}$ for each vertex and each pair of vertices. The equations are:

$$
\begin{cases}X_{1}+X_{2}+\ldots+X_{|V|}=(|V|+k) \cdot C & \\ X_{i}+X_{i}^{\prime}=3 \cdot C & \text { for every } i \in V \\ X_{i}+X_{j}+X_{i j}^{\prime \prime}=4 \cdot C & \text { for every }(i, j) \notin E\end{cases}
$$

If there is a clique of size $k$, then we will have a solution in every commutative finite semigroup (and even in the free commutative semigroup). We take $X_{i}=$ $2 \cdot C$ if the vertex $i$ is in the clique or $X_{i}=C$ if it isn't. First equation is satisfied, because there are exactly $k$ vertices in the clique. The equations of the second and third type can be satisfied by taking $X_{i}^{\prime}$ and $X_{i j}^{\prime \prime}$ equal to $C$ or $2 \cdot C$. When there is no edge $(i, j)$, then at most one of vertices $i$ and $j$ is in the clique, so it's possible to satisfy the equations of the third type.

When there is a solution in every commutative finite semigroup, then there is also some solution $\bar{x}$ in the following semigroup $S$ : Elements of $S$ are $\{1,2, \ldots, M\}$ for large enough $M=\max (5,|V|+k+1)$. The operation $a+b$ is defined as $\min (M, a+b)$. The coefficient $C$ evaluates to 1 . From equations of the second type we see that $x_{i}=1$ or 2 . We take vertex $i$ to a clique iff $x_{i}=2$. The equations of the third type guarantees that two vertices cannot be in the clique if there is no edge between them. First equation says that the clique has size $k$.

\section{Other Questions}

It may be interesting to check if a system has a solution in other classes of semigroups, for example all finite semigroups or all idempotent finite semigroups.

For idempotent finite semigroups this question is easy, as we know that there are only finitely many idempotent semigroups with a given number of generators (see [9]). Moreover all these semigroups can be listed, because there is a formula for maximum number of elements in such semigroup. It is enough to check if a system has a solution in all semigroups generated by our coefficients, which we can do one semigroup after another. For any semigroup, when there is a solution in a subsemigroup generated by coefficients, it is also a solution in whole semigroup. It may be interesting to find an algorithm with better complexity.

It remains open how to check that in class of all finite semigroups. It is not difficult to prove that in every finite semigroup $S$, there are elements $u, v$ such that the subsemigroup $\{u s v: s \in S\}$ is a two-sided ideal and a group. So there is a hope that (with more effort that for commutative case) the problem could be reduced to solving systems of equations over all finite groups. However unlike in the commutative case, there are systems of equations satisfied in all finite 
groups, but not in the free group [3]. So the reduction to finite groups do not give a solution yet.

It would be also interesting to know if the problem of checking if arbitrary first order formula is satisfied in every commutative finite semigroup is decidable.

\section{References}

1. Bojańczyk, M., Colcombet, T.: Tree-walking automata cannot be determinized. In: Díaz, J., Karhumäki, J., Lepistö, A., Sannella, D. (eds.) ICALP 2004. LNCS, vol. 3142, p. 246-256. Springer, Heidelberg (2004)

2. Borosh, I., Treybig, L.B.: Bounds on positive integral solutions of linear diophantine equations. Proc. Amer. Math. Soc. 55(2), 299-304 (1976)

3. Coulbois, T., Khelif, A.: Equations in free groups are not finitely approximable. Proc. Amer. Math. Soc. 127(4), 963-965 (1999)

4. Gurevich, Y.: The word problem for certain classes of semigroups. Algebra and Logic 5(5), 25-35 (1966) (in Russian)

5. Gutiérrez, C.: Satisfiability of word equations with constants is in exponential space. In: Foundations of Computer Science, pp. 112-120 (1998)

6. Karp, R.M.: Reducibility among combinatorial problems. In: Miller, R.E., Thatcher, J.W. (eds.) Complexity of Computer Computations, pp. 85-103. Plenum Press (1972)

7. Klima, O., Tesson, P., Therien, D.: Dichotomies in the complexity of solving systems of equations over finite semigroups. Theor. Comp. Sys. 40(3), 263-297 (2007)

8. Makanin, G.S.: The problem of solvability of equations in a free semigroup. USSR Sbornik 32, 129-198 (1977)

9. McLean, D.: Idempotent semigroups. The American Mathematical Monthly 61(2), 110-113 (1954)

10. Plandowski, W.: Satisfiability of word equations with constants is in pspace. In: Foundations of Computer Science, pp. 731-742 (1999)

11. Plandowski, W., Rytter, W.: Application of lempel-ziv encodings to the solution of word equations. In: International Colloquium on Automata, Languages and Programming, pp. 731-742 (1998)

12. Tarski, A., Mostowski, A., Robinson, M.R.: Undecidable theories. North-Holland, Amsterdam (1953) 